\title{
New and Emerging Infections: a Select Review of Evolving Pathogens
}

\author{
Hanan Haydar, $M D^{*}$ \\ Ethan Leonard, MD \\ Ankita Desai, MD
}

\author{
Address \\ *Department of Pediatric Infectious Diseases, University Hospitals Rainbow \\ Babies and Children's Hospital, 11100 Euclid Avenue, Mailstop RBC 6008 A, \\ Cleveland, OH, 44106, USA \\ Email: Hanan.haydar@uhhospitals.org
}

Published online: 17 May 2019

(C) Springer Nature Switzerland AG 2019

This article is part of the Topical Collection on Disaster Medicine

Keywords Emerging infections - Current treatment options - Zika virus - Ebola virus · Multidrug-resistant gram-negative pathogens $\cdot$ Candida auris

\begin{abstract}
Purpose of review Emerging infections are diseases that are either newly discovered in a population or previously known but increasing in incidence. We have chosen to review a few emerging infections that are most notable at this time with new treatment options. We separated them into three categories: viruses, bacteria, and fungi.

Recent findings Among emerging viral pathogens, we focused on Ebola virus where we have a monoclonal antibody that has been shown safe for treatment in adults, and promising vaccine candidates. Zika virus is also reviewed, although care focuses on symptomatic treatment, we now have several vaccines under development for prevention. Bacterial pathogens of concern are gram-negative bacteria with multidrug resistance. Current treatment options include: use of $\beta$-lactamase inhibitors, use of combination therapy, and use of novel non-antibiotic therapies. Lastly, Candida auris is a newly identified Candida species that can be highly resistant to routine antifungal therapy. A new agent, SCY-078, has shown growth inhibition and also antibiofilm activity against $C$. auris.

Summary In conclusion, while we have chosen to review only a few emerging infections, there are many others circulating and newer pathogens will arise. We need to develop, evaluate, and disseminate novel tools to identify and treat new and current emerging infections.
\end{abstract}


Emerging infections are diseases that are either newly discovered in a population or previously known but increasing in incidence. While there are many infectious disease threats encountered all over the world on a daily basis, we have chosen to review a few emerging infections that are most notable at this time with new treatment options on the horizon. We have separated them into three categories: viruses, bacteria, and fungi. Among emerging viral pathogens, we will focus on Ebola and Zika viruses. Bacterial pathogens of concern are the rapid emergence of gram-negative bacteria with multidrug resistance. Lastly, Candida auris is a newly identified Candida species that is rapidly spreading through the world and has the potential to be highly resistant to routine antifungal therapy.

\section{Viruses}

Zika virus Zika virus is a flavivirus, in the family Flaviviridae. Zika was first discovered in 1947 with the first reported case of human infection in 1952; subsequently, reports of sporadic infections followed [1]. The viral transmission cycle is human-mosquitohuman. After its discovery, the virus was isolated on several occasions from the Aedes africanus mosquito. Seroprevalence studies indicate that the virus was present in a broad geographic distribution in much of Africa and Southeast Asia, and, therefore, suggest that human infection was common.

Zika virus gained attention when it was first identified in the Americas in March of 2015 with an outbreak in Brazil. By December 2015, an estimated 1.3 million cases had been identified. As of March 2016, the virus had spread to at least 33 countries and territories in the Americas [2•].

It was not until the fall of 2015 when Brazilian investigators noted an increase in infants born with microcephaly in the areas that Zika virus was first reported that the implications of infection in pregnancy were understood. Substantial evidence indicates that Zika virus can be transmitted from mother to fetus during pregnancy. Zika virus RNA has been identified in the amniotic fluid of mothers whose fetuses had cerebral abnormalities detected by ultrasound, and viral antigen and RNA have both been identified in brain tissue and placenta of infants born with microcephaly, and also tissues from miscarriages. In addition to vertical transmission, it can also be transmitted sexually, and through blood or tissue donation.

Diagnosis can be made by detection of viral nucleic acid by RT-PCR and/or the detection of IgM antibodies. Diagnosis by RT-PCR is possible within 1 week of symptoms. Viral RNA has been detected in the serum of pregnant women whose fetus had evidence of congenital infection for approximately 10 weeks after infection. IgM typically appears within the first week of symptoms and will persist for several months. Recommended laboratory testing for possible congenital Zika virus includes testing for Zika virus RNA in serum and urine, as well as Zika IgM antibodies in serum [2•].

Zika virus infection during pregnancy can cause serious birth defects including structural abnormalities in the brain and eye. Clinical evaluation of infants born with findings consistent with congenital Zika infection includes Zika laboratory testing as listed above, developmental screening, ultrasound or MRI of the head, comprehensive ophthalmology evaluation, and hearing screen [3].

The U.S. Zika Pregnancy and Infant Registry was created to follow all pregnancies with laboratory evidence of confirmed or possible Zika virus infection and infants resulting from these pregnancies. One in seven children evaluated had a Zika-associated birth defect and/or a neurodevelopmental abnormality possibly associated with congenital Zika virus infection. Early identification and intervention for adverse neurodevelopmental outcomes are needed to improve cognitive, social, and behavioral functioning [3].

Unfortunately, treatment is supportive for symptoms as there is no specific antiviral therapy currently available. In light of global trends towards urbanization, climate change, and intercontinental travel, research for broad-spectrum antiviral medications against Flaviviruses are underway. Potential targets include viral proteins, such as viral protease or polymerases, and host targets that are exploited by the virus for entry and replication, such as those needed for nucleoside biosynthesis [4]. Given the severe consequences of infection during pregnancy, there is a great urgency to develop an effective vaccine. A highly safe, efficacious, and economical vaccine is needed to decrease vertical transmission in susceptible pregnant women in the developing countries with the highest Zika burden. Several vaccine candidates including purified inactivated virus, live attenuated virus, virus- like particles, DNA, modified RNA, 
viral vectors, and subunit vaccines are all under development, with some in clinical trials [5]. Tremendous progress has been made in drug and vaccine development against Zika virus. However, until there is a viable option for either treatment or vaccination, other preventative measures are paramount.

Prevention and control measures center on avoiding mosquito bites, reducing sexual or blood transmission, and controlling the mosquito vector. Pregnant women are encouraged to avoid areas with ongoing Zika virus transmission, unprotected sexual contact with partners at risk for Zika virus infection, and to use methods to avoid mosquito bites such as insect repellent, permethrin treatment of clothes, bed nets, window screens, and air conditioning [2•]. Couples who have possible Zika exposure and are considering pregnancy should postpone pregnancy for 4 months following potential exposure or diagnosis of Zika. Pregnant women who report illness consistent with Zika virus within 2 weeks of returning from an area with Zika transmission should be tested for Zika virus.

Ebola virus Ebola virus disease is a rare and deadly infection caused by a group of viruses in the genus Ebolavirus. Four species are responsible for human disease: Zaire, Sudan, Tai Forest, and Bundibugyo. The virus was first discovered in 1976 near the Ebola River in the Democratic Republic of Congo. The virus has caused outbreaks from time to time, but the largest outbreak of Ebola virus disease spread over West Africa starting in 2014. The three most affected countries were Guinea, Liberia, and Sierra Leone. There were more than 28,000 cases and 11,000 deaths [6]. The outbreak was successfully controlled in 2016 with the implementation of effective and coordinated public health measures that involved rapid identification, isolation of cases, contact tracing, and isolation of contacts. Investigational studies during this outbreak have led to progress in the development of biologics and medications to treat this deadly disease [7]. Despite these new tools, the pillars of control must remain. Re-emergence of disease in 2017 and 2018 outbreaks in the Democratic Republic of Congo highlight the need for continued vigilance. Efforts to develop an effective vaccine must continue [8].

Following initial infection, the disease spreads rapidly presenting with an acute febrile illness and high-level viral replication, followed by severe immune suppression, sepsis, organ failure, and death. A broad range of clinical signs and symptoms are noted with fever, headache, malaise, nausea, vomiting, diarrhea, hypotension, and bleeding all reported. Major clinical disease is due to gastrointestinal tract dysfunction including electrolyte abnormalities. Due to rapid disease progression, treatment requires early aggressive supportive care with rehydration, antipyretics, and intensive care. Careful clinical management to correct fluid loss and hypotension are needed to prevent multisystem organ failure [9•]. A meta-analysis of 20 Ebola virus disease outbreaks showed an estimated case-fatality rate of $65.4 \%$ [9•].

A number of therapeutic agents have been tried under compassionate use in the treatment of individuals with Ebola virus disease. Monoclonal antibody, biologics, and antivirals are all under development. Antiviral favipiravir has been shown to be efficacious in treating animals infected with aerosolized E718 strain of Ebola virus. The drug induced a viral mutagenesis that leads to reduced viral infectivity and replication [9•]. ZMapp is a novel immunotherapeutic which is a combination of monoclonal antibodies. It is a cocktail of three chimeric, monoclonal antibodies that target the Ebola virus glycoprotein core. In a randomized, controlled trial, it did not meet statistical criteria for efficacy, it did appear to be beneficial [10 $\bullet$. It is now considered to be a component of the standard of care.

Two vaccine candidates are nearing consideration for licensure. These include the V920 vaccine and the Ad26ZEBOV/MVA-BN-Filo prime-boost vaccine; both vaccines produce an immune response that is sustained for up to 1year post immunization [11]. In one study, the V920 vaccine was given to patient contacts, their contacts, front-line workers, and health-care workers to provide a ring of vaccine-elicited protection [12].

Other containment strategies under development include improved ways to provide care for infected individuals, such as biosecure cubicles which allow for providing intravenous fluids and therapeutics through specialized ports. In order to overcome this very deadly disease, novel research must continue to explore the development of both preventative and therapeutic modalities.

\section{Bacteria}

Multidrug-resistant gram-negative pathogens Antimicrobial resistance (AMR) is a complicated and longstanding problem since the twentieth century. However, AMR is now considered an alarming public health crisis by the 
scientific and political community due to the evolution of resistant microbes and relative lag in the development of new antibiotics. Starting with the emergence of grampositive bacterial resistance as penicillin and methicillinresistance in Staphylococcus aureus to vancomycin-resistant Enterococci, we are now facing a rise in multidrugresistant gram-negative (MDRGN) infections. Since the late 1990s, some infections have become virtually untreatable, and this phenomenon has been reported worldwide. Significant morbidity and mortality are attributed to MDRGN infections, not only in adults but also in infants and children [13]. Some experts consider the spread of resistant gram-negative infections as the most pressing emerging issue in bacterial resistance [14]. In this review, we present a brief overview of emerging resistance in gram-negative bacteria.

The definition of multidrug resistance varies between countries [15•]. The following definitions have been developed by an international panel of experts through a joint initiative by the Centers for Disease Control and Prevention (CDC) and the European Centre for Disease Prevention and Control (ECDC). Multidrug resistance (MDR) is an acquired non-susceptibility to at least one agent in three or more antimicrobial classes. Extensively drug resistance (XDR) is defined as non-susceptibility to at least one agent in all, but two or fewer antimicrobial classes. Pandrug resistance is non-susceptibility to all agents in all available antimicrobial classes [16].

Gram-negative bacteria become MDR in multiple ways. Intrinsic mechanisms of resistance include the presence of efflux pumps that are encoded on a chromosomal gene and limit antibiotic levels intracellularly. Acquired mechanisms of resistance include the transfer of resistance genes horizontally mostly by plasmidmediated conjugation or spontaneous mutation in existing genes. Mechanisms of resistance may either be enzymatic such as the production of $\beta$-lactamases or non-enzymatic such as modification of the components

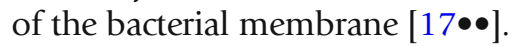

The Ambler system is a classification system for $\beta$ lactamases. This taxonomy includes 4 classes (A, B, C, and D); classification depends on amino acid sequences and their active site. Class A enzymes include the extended-spectrum B-lactamases (ESBLs) and Klebsiella pneumoniae carbapenemase (KPC) enzymes; class B enzymes are metallo- $\beta$-lactamases. Class $\mathrm{C}$ enzymes are cephalosporinases (AmpC). Class D are plasmidencoded oxacillinase (OXA-48) enzymes [14].

Unfortunately, many MDRGN bacteria not only possess $\beta$-lactam resistance, they often show resistance to multiple classes of antibiotics. Plasmids often carry multiple antibiotic-resistant genes. For example, some enzymes modify targets for antibiotics (e.g., ribosomal RNA-methylation leading to aminoglycoside-resistance), or the antibiotics themselves (e.g., acetyltransferases that modify quinolones and aminoglycosides). Another resistance mechanism is efflux pumps that are encoded by plasmids. These pumps extrude $\beta$ lactam antibiotics as well as quinolones, aminoglycosides, and tetracycline [14].

Physicians should be concerned about MDRGN infections for multiple reasons. Fears of a post-antibiotic era may eventually become a reality. Some infections with carbapenemase-producing Pseudomonas aeruginosa, Klebsiella pneumoniae, and Acinetobacter baumannii are not able to be treated with standard antimicrobial options. MDRGN infections are associated with increased health-care cost, morbidity, mortality, and duration of hospitalization [14]. A meta-analysis showed a twofold increased risk of allcause-hospital mortality in patients with MDR Pseudomonas aeruginosa as compared with patients with susceptible $P$. aeruginosa [18]. Lastly, resistance can spread widely across a microbiologic level. Gram-negative bacteria have plasmids that often carry genes causing the resistance; these plasmids are easily shared among other bacteria or within species within the host or environment [13].

With increased international travel and medical tourism, we are encountering more MDRGN outbreaks on a regional level as well as worldwide [19]. The presence of New Delhi metallo-beta-lactamase (NDM) enzyme is endemic in the Indian sub-continent, Arabian Peninsula, North Africa, and the Balkan States. Bacterial strains are now disseminated into the community and found in drinking water [20]. Sporadic outbreaks have been also reported in the USA. Klebsiella pneumoniae carbapenemases (KPC) are currently endemic in certain Latin American countries, parts of the USA, and some Mediterranean countries [14]. These MDRGN bacteria spread in silence and pose a major challenge to infection control. As Enterobacteriaceae are part of normal gut microbiota, people may be colonized asymptomatically and serve as reservoirs for spread to others.

Treatment in adults and children is often complicated and often requires consultation with an infectious disease specialist. The emergence of MDRGN infections led to the re-discovery of older antibiotic classes and to the development of new agents. For invasive ESBL- and Amp C-producing bacteria, the treatment of choice is often carbapenems. Treatment options are now more limited for carbapenemase-producing gram-negative 
bacilli because of the emerging resistance to older agents such as polymyxins (colistin or polymyxin B), tigecycline, and fosfomycin. Also, these agents have unfavorable pharmacokinetic profiles and toxicity [21•]. Tigecycline is a bacteriostatic agent that has poor serum and urine levels, limiting its use in the urinary tract and bloodstream infections. The drug has no activity against Pseudomonas, Proteus, Morganella, and Providencia. Nephrotoxicity and neurotoxicity limit the use of polymyxins. This class has limited activity against Proteus, Providencia, Morganella, and Serratia. Fosfomycin and nitrofurantoin clinical use is limited to urinary tract infections [14]. Limitations of using these older agents along with emerging resistance fueled the discovery of new agents.

New $\beta$-lactamase inhibitors such as avibactam (discovered in 2011) and vaborbactam (approved 2017) have been used in combination with $\beta$-Lactam antibiotics such as ceftazidime, aztreonam, or meropenem. Ceftazidime-avibactam was approved by the FDA in 2015. The avibactam inhibits Ambler class A, C, and some of class D. Ceftazidime-avibactam is approved for the treatment of complicated intra-abdominal infections, urinary tract infections, and most recently ventilator/hospital-acquired pneumonia [21•, 22•]. Meropenem/vaborbactam acts on Ambler class A and C enzymes (mostly KPC). This combination is approved

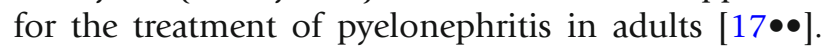
Currently, at least 4 new $\beta$-lactam/ $\beta$-lactamase inhibitors (BLI) and one new monobactam/BLI are in the early stages of clinical development; these compounds demonstrated potent in vitro and in vivo efficacy against MDRGN bacteria in preclinical trials. These therapeutics include cefepime/zidebactam, nacubactam, cefepime/ AAI101, aztreonam/avibactam, ceftaroline/avibactam,

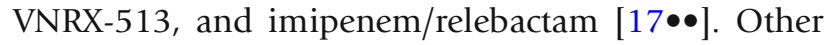
novel antibiotics used for the treatment of MDRGN bacteria include newer aminoglycosides, but their use has been limited due to the emergence of resistance and narrow safety profile $[22 \bullet, 23]$. Plazomicin is a new aminoglycoside approved in 2017 specifically designed to overcome CRE strains that have aminoglycosidemodifying enzymes. Currently, resistance to plazomicin is already reported [22•]. Other non-BL/BLI agents which are now in clinical development include murepavadin, finafloxacin, omadacycline, and eravacycline $[17 \bullet \bullet]$.

The continuing resistance to new classes of antibiotics led to advanced consideration for combination antibiotic therapy. Combination therapy through its synergistic effect increases bacterial killing and decreases the emergence of resistant isolates. Also, throughout the years, combination therapy has been effective against other hard to treat infections such as mycobacterial, viral, and parasitic infections (HIV, tuberculosis, and

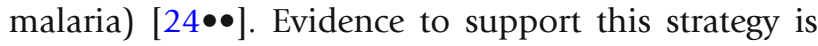
mixed and is based on retrospective or nonrandomized prospective cohort studies $[25 \bullet \bullet, 26]$. These studies favor combination therapy over monotherapy for CRE, despite increased cost and toxicity. Two instances showed that combination therapy worked: (a) for isolates with MIC of $<16 \mathrm{mg} / \mathrm{L}$ for meropenem or imipenem [27•]; (b) for patients with a high mortality score and CRE bloodstream infections [28•]. Ceftazidime-avibactam has activity against KPCs but not NDM; however, combined with aztreonam, this drug has potent activity against NDM [14].

Due to the rapid emergence of multidrug resistance, research is underway for the development of novel non-antibiotic therapies. These strategies include antivirulence strategies which target [1]: toxins/ secretion systems (e.g., Shigamab, a monoclonal antibody that targets Stx-1 and Stx- 2 produced by Escherichia coli is now in phase 2 clinical trial) [2•], biofilms and adherence [3], signaling and regulation. Another promising development is phage therapy which offers multiple advantages over antibiotics. This therapy will not affect normal microbial flora or human cells, as they are only specific for pathogenic bacteria. Significant limitations and barriers do exist, however, such as stability issues, development of resistance, and host immune response. Since phage therapy targets specific strains, multiple phages are required to target strains within a species. Phage cocktails have been successfully tested in humans with phase 1 and 2 clinical trials for efficacy against Pseudomonas chronic

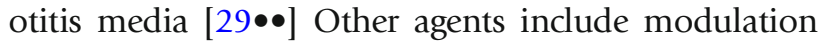
of host immunity and the microbiome. Examples of immune modulation include innate defense regulator peptides and agonists of innate immune components such as toll-like receptors and NOD-like receptors. They work by enhancing innate immune mechanisms to help overcome infection; they can be used alone or in combination with systemic antibiotics. One limitation to these agents is they can lead to potentially harmful inflammation [30]. Examples of modulating the host microbiome is the use of fecal microbiota transplant in the treatment of recurrent Clostridium difficile infection. To date, this is the most successful use of modulation of the human microbiome as a

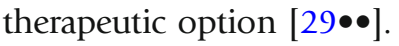


Because few studies exist addressing the treatment of MDRGN bacteria in the pediatric population, data are limited. Only ceftazidime-avibactam has been approved in pediatric patients. For the time being, we must rely on adult literature, expert opinion, and observational studies [13].

Other strategies have been implemented to control emerging resistance and stop the crisis. These include the following: implementing antimicrobial stewardship, enhanced surveillance by reporting MDR bacteria, and screening for high-risk patients with a detailed history including hospital stay in last 12 months, travel history, prolonged hospitalization, and family member with MDR infection. Infection control measures include strict contact isolation with identified infections, safe decontamination practices, and robust cleaning protocols. Finally, patient education is a crucial part in preventing the spread of bacterial resistance. These measures may include showing patients and their families effective hand washing measures, appropriate hygiene and toilet use, and proper disposal of bodily fluids [15•].

\section{Fungal disease}

Candida auris Candida auris is an emerging multidrugresistant yeast. The yeast was first described in 2009 after the discovery from an external ear canal in a patient in Japan. This fungus phenotypically resembles another Candida species, Candida haemulonii, and often needs molecular methods for accurate identification. C. auris often exhibit resistance to fluconazole, with variable susceptibilities to other azoles, amphotericin B, and echinocandins. Resistance rates to amphotericin B have been reported to be 30-40\% and $5-10 \%$ to echinocandins. Half of all isolates are noted to be resistant to two or more antifungals and a small percentage are resistant to all currently available antifungals [31•].

Researchers have yet to understand why Candida auris rapidly emerged in the past 10 years and why it has spread worldwide. It is reported to cause serious invasive infection and is associated with a high mortality rate, over 60\%. Interestingly, whole genome analysis suggests a near-simultaneous emergence of $C$. auris in multiple locations throughout the world, rather than a rapid spread from a single source. Phylogenetic analysis showed four distinct clades separated by over tens of thousands of single nucleotide polymorphisms representing distinct geographic regions (South Asia, South Africa, and South America, and East Asia). However, isolates within each regional cluster showed very low genetic diversity [31•]. It has been postulated that increased use of antifungals and antifungal selection pressure may be responsible for its rapid emergence [32].

Antifungal selection pressure may be responsible for its emergence, but there are a number of other reasons that may explain how Candida auris has spread rapidly among institutions. First, difficulties in accurately identifying the species by commercial methods may lead to delayed diagnosis with a consequent delay in the implementation of appropriate infection control precautions. Candida auris persists in hospital environments and has been detected on bed linens, windowsills, tables, equipment, monitors, and floors. The pathogen is not eradicated by routine cleaning methods and needs more aggressive terminal cleaning using cleaners active against C. difficile spores [33]. Controlling this emerging pathogen relies heavily on rapid and accurate identification to lead to rapid isolation of infected or colonized patients. Until more effective treatment options are readily available, we must maintain strict infection control precautions to prevent the spread of infection.

Due to a significant resistance to multiple commonly used antifungals, the development of new agents effective against Candida auris has been pursued. One new agent, SCY-078, a new orally bioavailable 1,3-B-D-glucan synthesis inhibitor, has potent activity against $C$. auris. It has shown growth inhibition and also antibiofilm activity [34].

In conclusion, while we have chosen to review only a few emerging infections facing our population, there are many others already circulating and newer pathogens will arise. The importance of prevention is paramount as evidenced by recurrent outbreaks of Ebola virus and the rapid spread of Candida auris. Early identification of infection with rapid implementation of infection control precautions must be a priority. As we have shown with emerging gram-negative bacterial resistance, many new treatment options are now in the pipeline. However, it currently takes years for new drugs to navigate through the approval process. Given the rapidity of emerging resistance, accelerating discovery and streamlining approval are critical. 


\section{Compliance with Ethical Standards}

\section{Conflict of Interest}

Hanan Haydar declares that she has no conflict of interest. Ethan Leonard declares that he has no conflict of interest. Ankita Desai declares that she has no conflict of interest.

Human and Animal Rights and Informed Consent

This article does not contain any studies with human or animal subjects performed by any of the authors.

\section{References and Recommended Reading}

Papers of particular interest, published recently, have been highlighted as:

- Of importance

$\bullet \quad$ Of major importance

1. das Neves Almeida R, Racine T, Magalhaes KG, Kobinger GP. Zika virus vaccines: challenges and perspectives. Vaccines. 2018;6(3).

2. $\quad$ Petersen LR, Jamieson DJ, Honein MA. Zika virus. N Engl J Med. 2016;375(3):294-5.

Comprehensive review of Zika virus epidemiology and clinical manifestations.

3. Rice ME, Galang RR, Roth NM, Ellington SR, Moore CA, Valencia-Prado M, et al. Vital signs: Zika-associated birth defects and neurodevelopmental abnormalities possibly associated with congenital Zika virus infection - U.S. Territories and Freely Associated States, 2018. MMWR Morb Mortal Wkly Rep. 2018;67(31):858-67.

4. Boldescu V, Behnam MAM, Vasilakis N, Klein CD. Broad-spectrum agents for flaviviral infections: dengue, Zika and beyond. Nat Rev Drug Discov. 2017;16(8):565-86.

5. Garg H, Mehmetoglu-Gurbuz T, Joshi A. Recent advances in Zika virus vaccines. Viruses. 2018;10(11).

6. Bell BP, Damon IK, Jernigan DB, Kenyon TA, Nichol ST, O'Connor JP, et al. Overview, control strategies, and lessons learned in the CDC response to the 2014-2016 Ebola epidemic. MMWR Supplements. 2016;65(3):411.

7. Damon IK, Rollin PE, Choi MJ, Arthur RR, Redfield RR. New tools in the Ebola arsenal. N Engl J Med. 2018;379(21):1981-3.

8. Levy Y, Lane C, Piot P, Beavogui AH, Kieh M, Leigh B, et al. Prevention of Ebola virus disease through vaccination: where we are in 2018. Lancet. 2018;392(10149):787-90.

9. $\quad$ Kilgore PE, Grabenstein JD, Salim AM, Rybak M. Treatment of Ebola virus disease. Pharmacotherapy. 2015;35(1):43-53.

Good review of Ebola virus disease and management options.
10. Davey RT Jr, Dodd L, Proschan MA, Neaton J, Neuhaus Nordwall J, Koopmeiners JS, et al. A randomized, controlled trial of ZMapp for Ebola virus infection. $\mathrm{N}$ Engl J Med. 2016;375(15):1448-56.

This study detailed the use of ZMapp for Ebola virus infection, though not statistically significant, it did appear to be beneficial.

11. Espeland EM, Tsai CW, Larsen J, Disbrow GL. Safeguarding against Ebola: vaccines and therapeutics to be stockpiled for future outbreaks. PLoS Negl Trop Dis. 2018;12(4):e0006275.

12. Henao-Restrepo AM, Camacho A, Longini IM, Watson $\mathrm{CH}$, Edmunds WJ, Egger $\mathrm{M}$, et al. Efficacy and effectiveness of an rVSV-vectored vaccine in preventing Ebola virus disease: final results from the Guinea ring vaccination, open-label, cluster-randomised trial (Ebola Ca Suffit!). Lancet. 2017;389(10068):505-18.

13. Hsu AJ, Tamma PD. Treatment of multidrug-resistant gram-negative infections in children. Clin Infect Dis. 2014;58(10):1439-48.

14. Vasoo S, Barreto JN, Tosh PK. Emerging issues in gramnegative bacterial resistance: an update for the practicing clinician. Mayo Clin Proc. 2015;90(3):395-403.

15. Exner M, Bhattacharya S, Christiansen B, Gebel J, Goroncy-Bermes P, Hartemann P, et al. Antibiotic resistance: what is so special about multidrug-resistant gram-negative bacteria? GMS hygiene and infection control. 2017;12:Doc05.

This is a summary about MDRGN infections, infection control measures, and newly implemented strategies to decrease spread of these infections.

16. Magiorakos AP, Srinivasan A, Carey RB, Carmeli Y, Falagas ME, Giske CG, et al. Multidrug-resistant, extensively drug-resistant and pandrug-resistant bacteria: an international expert proposal for interim standard 
definitions for acquired resistance. Clin Microbiol Infect. 2012;18(3):268-81.

17.• Avery LM, Nicolau DP. Investigational drugs for the treatment of infections caused by multidrug-resistant gram-negative bacteria. Expert Opin Investig Drugs. 2018;27(4):325-38.

This excellent review summarizes in vitro and in vivo efficacy studies, as well as available clinical trials for new agents in development for treatment of MDRGN infections.

18. Nathwani D, Raman G, Sulham K, Gavaghan M, Menon V. Clinical and economic consequences of hospital-acquired resistant and multidrug-resistant Pseudomonas aeruginosa infections: a systematic review and meta-analysis. Antimicrob Resist Infect Control. 2014;3(1):32.

19. Munoz-Price LS, Poirel L, Bonomo RA, Schwaber MJ, Daikos GL, Cormican M, et al. Clinical epidemiology of the global expansion of Klebsiella pneumoniae carbapenemases. Lancet Infect Dis. 2013;13(9):78596.

20. Walsh TR, Weeks J, Livermore DM, Toleman MA. Dissemination of NDM-1 positive bacteria in the New Delhi environment and its implications for human health: an environmental point prevalence study. Lancet Infect Dis. 2011;11(5):355-62.

21. Sherry N, Howden B. Emerging gram negative resistance to last-line antimicrobial agents fosfomycin, colistin and ceftazidime-avibactam - epidemiology, laboratory detection and treatment implications. Expert Rev Anti-Infect Ther. 2018;16(4):289-306.

In this review, the authors highlight resistance to three of the most important last-line antimicrobials for treatment of MDR and XDR gram-negatives: fosfomycin, colistin, and ceftazidime-avibactam.

22. Petty LA, Henig O, Patel TS, Pogue JM, Kaye KS. Overview of meropenem-vaborbactam and newer antimicrobial agents for the treatment of carbapenemresistant Enterobacteriaceae. Infect Drug Resist. 2018;11:1461-72.

Comprehensive detailed review of meropenem-vaborbactam, an antibiotic recently approved for treatment of UTI and acute pyelonephritis.

23. Aggen JB, Armstrong ES, Goldblum AA, Dozzo P, Linsell MS, Gliedt MJ, et al. Synthesis and spectrum of the neoglycoside ACHN-490. Antimicrob Agents Chemother. 2010;54(11):4636-42.

24.• El Chakhtoura NG, Saade E, Iovleva A, Yasmin M, Wilson B, Perez F, et al. Therapies for multidrug resistant and extensively drug-resistant non-fermenting gram-negative bacteria causing nosocomial infections: a perilous journey toward 'molecularly targeted' therapy. Expert Rev Anti-Infect Ther. 2018;16(2):89-110. A excellent detailed summary covering mechanisms of resistance of four major groups of non-fermenting gram-negative bacilli. They also cover promising novel therapeutic options, particularly combination antibiotic strategies.
25.•• Perez F, El Chakhtoura NG, Papp-Wallace KM, Wilson BM, Bonomo RA. Treatment options for infections caused by carbapenem-resistant Enterobacteriaceae: can we apply "precision medicine" to antimicrobial chemotherapy? Expert Opin Pharmacother. 2016;17(6):761-81.

Detailed review of pharmacotherapy in treating CRE infections and new promising treatments.

26. Paul M, Carmeli Y, Durante-Mangoni E, Mouton JW, Tacconelli E, Theuretzbacher U, et al. Combination therapy for carbapenem-resistant gramnegative bacteria. J Antimicrob Chemother. 2014;69(9):2305-9.

27. Machuca I, Gutierrez-Gutierrez B, Gracia-Ahufinger I, Rivera Espinar F, Cano A, Guzman-Puche J, et al. Mortality associated with bacteremia due to colistinresistant Klebsiella pneumoniae with high-level Meropenem resistance: importance of combination therapy without Colistin and Carbapenems. Antimicrob Agents Chemother. 2017;61(8).

This is a prospective cohort study including episodes of bacteremia caused by colistin-resistant and high-level meropenem-resistant KPC-producing K. pneumoniae showing that combination therapy reduces mortality.

28. Gutierrez-Gutierrez B, Salamanca E, de Cueto M, Hsueh PR, Viale P, Pano-Pardo JR, et al. Effect of appropriate combination therapy on mortality of patients with bloodstream infections due to carbapenemase-producing Enterobacteriaceae (INCREMENT): a retrospective cohort study. Lancet Infect Dis. 2017;17(7):726-34.

A retrospective cohort study with patients with clinically significant monomicrobial bloodstream infections due to CPE recruited from 26 tertiary hospitals in ten countries. This study showed that combination therapy was associated with improved survival only in patients with a high mortality score.

29.• Hauser AR, Mecsas J, Moir DT. Beyond antibiotics: new therapeutic approaches for bacterial infections. Clin Infect Dis. 2016;63(1):89-95.

An excellent review about new non-antibiotic aproaches for bacterial infections, including targeting of bacterial virulence factors, utilization of bacteriophages to kill bacteria, and manipulation of the microbiome to combat infections.

30. Hancock RE, Nijnik A, Philpott DJ. Modulating immunity as a therapy for bacterial infections. Nat Rev Microbiol. 2012;10(4):243-54.

31. Lockhart SR, Etienne KA, Vallabhaneni S, Farooqi J, Chowdhary A, Govender NP, et al. Simultaneous emergence of multidrug-resistant Candida auris on 3 continents confirmed by whole-genome sequencing and epidemiological analyses. Clin Infect Dis. 2017;64(2):134-40.

Interesting article detailing emergence of $C$. auris in multiple continents across the world. Whole genome sequencing showed clades were grouped by geographic region. 
32. Jeffery-Smith A, Taori SK, Schelenz S, Jeffery K, Johnson EM, Borman A, et al. Candida auris: a review of the literature. Clin Microbiol Rev. 2018;31(1).

33. Sears D, Schwartz BS. Candida auris: an emerging multidrug-resistant pathogen. Int J Infect Dis. 2017;63:95-8.

34. Larkin E, Hager C, Chandra J, Mukherjee PK, Retuerto $\mathrm{M}$, Salem I, et al. The emerging pathogen Candida auris: growth phenotype, virulence factors, activity of antifungals, and effect of SCY-078, a novel glucan synthesis inhibitor, on growth morphology and biofilm formation. Antimicrob Agents Chemother. 2017;61(5).

\section{Publisher's Note}

Springer Nature remains neutral with regard to jurisdictional claims in published maps and institutional affiliations. 